\title{
Der Adnexbefund
}

Kommission Qualitätssicherung der Schweizerischen Gesellschaft für Gynäkologie und Geburtshilfe

La version française suivra

La version française suivra

\section{Präambel}

Aufbauend auf Evidence-based Medicine (EBM) sollen Guidelines eine Hilfeleistung und Orientierung für Diagnostik und Therapie an der individuellen Patientin erbringen. Die FMH legt Wert darauf, dass Guidelines insbesondere für die Ärzte in der Praxis, aber auch für den klinischen Bereich ihre Anwendung finden sollen. «Leitlinien» sind systematisch entwickelte Darstellungen und Empfehlungen mit dem Zweck, Arzt und Patientin bei der Entscheidung über zweckdienliche Massnahmen der Krankenversorgung (Prävention, Diagnostik, Therapie und Nachbehandlung) unter spezifischen klinischen Umständen zu unterstützen. Leitlinien stellen den Stand des Wissens über effektive und zweckdienliche Krankenversorgung zum Zeitpunkt der Veröffentlichung dar und wenden sich in erster Linie an den ärztlichen Bereich, erst sekundär an die Öffentlichkeit und die Versicherer. Wegen der Fortschritte der wissenschaftlichen Erkenntnisse müssen Leitlinien periodisch überarbeitet werden. Die Entscheidung darüber, ob einer bestimmten Empfehlung gefolgt werden soll, muss vom Arzt unter Berücksichtigung der bei der individuellen Patientin vorliegenden Gegebenheiten und der verfügbaren Ressourcen entschieden werden. Im Einzelfall müssen somit die aktuelle Situation der Patientin, ihr Umfeld, ihre sozioökonomische Situation, die Komorbidität und Nebendiagnosen, aber auch ihre ethische und religiöse Haltung mitberücksichtigt werden. Damit ist ein entscheidender ärztlicher Freiraum gegeben, welcher es überhaupt ermöglicht, Leitlinien in die Praxis umzusetzen. «Therapeutische Freiheit» bedeutet, dass der Arzt sowohl die Kompetenz hat wie auch die Verantwortung dafür trägt, dass die von ihm an der Patientin vorgenommene Diagnostik bzw. durchgeführte Therapie dem aktuellen Stand der Wissenschaft entspricht - therapeutische Freiheit somit gleichermassen als Freiheit wie auch als Verpflichtung, das Richtige zu tun.

Das Erstellen und die laufende Aktualisierung von Leitlinien erfordern hohe Kompetenz, Beharrungsvermögen und zeitlichen Einsatz. Vieles im Medizinberuf basierte schon immer auf Evidenz, neu sind eher die Methoden, Fortschritte und Kriterien, die eine Beurteilung der Validität von Studien und eine verständliche
Darstellung ihrer Resultate ermöglichen. Eine gute Leitlinie zeigt unter anderem auf, für welche Situationen sie Handlungsempfehlungen anbietet, wie aktuell die Handlungsanweisung ist, welche Grade der wissenschaftlichen Sicherung für jede einzelne Aussage besteht und inwiefern ihre Befolgung der Patientin voraussichtlich einen Nutzen bringen wird. Gute Leitlinien werden den Diskurs zwischen Medizin und Recht (decision making process) verbessern.

\section{Experten}

Dr. med. J. F. Delaloye, (SGGG), Koordinator und Leiter der Expertengruppe; Prof. Dr. med. E. Dreher (SGGG), Dr. med. M. Eberhard (SGGG), PD Dr. med. M. Fehr (SGGG), Prof. Dr. med. U. Haller (SGGG), PD Dr. med. O. Köchli (SGGG), Prof. Dr. med. J. Torhorst (SGP).

\section{Fachgruppen}

Schweizerische Gesellschaft für Gynäkologie und Geburtshilfe (SGGG), Schweizerische Gesellschaft für Pathologie (SGP).

\section{Einführung}

\subsection{Relevanz des Themas}

Adnexbefunde sind von klinischer Bedeutung und sind entweder genitalen Ursprungs (vom Ovar, von der Tube, paratubär oder vom Uterus) oder haben einen extragenitalen Ursprung (vom Darm oder extraperitoneal). Jedes Jahr werden in der Schweiz 620 neue Ovarialmalignome diagnostiziert. Dies entspricht 18 Krebsfällen pro 100000 Frauen. Meistens sind die Frauen bei Auftreten von Ovarialmalignomen zwischen 60 und 80 Jahre alt; jedoch ist eine von sechs Frauen unter 50 Jahren betroffen [1].

Ein Adnexbefund kann entweder einer funktionellen Zyste (Follikelzyste oder Gelbkörperzyste) entsprechen oder eine organische Zyste (Endometriom) bzw. einen eigentlichen Tumor (benigne, Borderline oder maligne) darstellen.

Ein tubärer oder paratubärer Befund kann folgenden Veränderungen entsprechen: embryonale Reste, Extrauteringravidität, Abszess, Sactosalpinx, Tumor. Ein parauteriner Befund entspricht oft einem Myom. Beispiele für extragenitale Befunde sind Skybala oder eine Beckenniere. 


\subsection{Ziel der Empfehlungen}

Die Empfehlungen enthalten alle relevanten Möglichkeiten der Diagnostik und Behandlungskonzepte. Die Malignome und prämalignen Veränderungen sollten damit erkannt werden. Darüber hinaus soll auch die Rate von unnützen Operationen bei funktionellen Zysten und Retentionszysten gesenkt werden. [2, 3].

\subsection{Methode}

Die Kommission Qualitätssicherung der SGGG hat eine Arbeitsgruppe aus Ärzten gebildet, welche in Universitätskliniken oder in Regionalspitälern arbeiten. Die vorliegenden Guidelines basieren auf einer Literaturrecherche, welche mit Hilfe der Medline-Datenbank für den Zeitraum 1988-2002 durchgeführt wurde. Die Aussagen wurden entsprechend der Klassifikation der Canadian Task Force on the Periodic Health Examination [4] beurteilt, klassifiziert und ausgewertet. ( $\varepsilon 1, \varepsilon 2, \varepsilon 3$ oder $\varepsilon 4)$. Der Evidenzgrad $\varepsilon 5$ wird nicht speziell vermerkt (siehe Kasten).

Nach Verabschiedung durch die Kommission Qualitätssicherung wurden die Leitlinien während 3 Monaten von 58 Ärzten/Ärztinnen für Frauenheilkunde sowie Allgemeinmedizin im klinischen Test überprüft und schliesslich unter Berücksichtigung dieser Erfahrungswerte dem Vorstand der SGGG zur Verabschiedung und Weiterleitung an die Guidelineskommission der Verbindung der Schweizerischen Ärztinnen und Ärzte FMH unterbreitet.

\section{Grad der Evidenz (Canadian Task Force on the Periodic Health} Examination) [4]

Grad I ( $\mathcal{E} 1)$ : Die Evidenz ist aufgrund randomisierter kontrollierter Studien (oder Meta-Analysen) von genügendem Umfang derart, dass die Gefahr, dass sie falsch positive oder falsch negative Resultate beinhalten, gering ist.

Grad II (ع2): Die Evidenz $\varepsilon 2$ basiert auf randomisierten, kontrollierten Studien, welche jedoch zu klein sind, um ihnen Grad I zuzusprechen; sie können positive Trends, welche jedoch statistisch nicht signifikant sind, oder gar keine Trends zeigen. Sie sind mit einem hohen Risiko falsch negativer Resultate verbunden.

Grad III (ع3): Die Evidenz $\varepsilon 3$ basiert auf nicht randomisierten Kontroll- oder Kohortenstudien, Fallserien, Fallkontrollstudien oder Querschnittstudien.

Grad IV (ع4): Die Evidenz $\varepsilon 4$ basiert auf der Meinung angesehener Experten oder Expertengremien, wie sie in publizierten Konsensuskonferenzen oder in Guidelines angegeben werden.

Grad V (ع5): Die Evidenz $\varepsilon 5$ basiert auf der Meinung derjenigen Personen, welche diese Guidelines geschrieben oder aktualisiert haben, beruhend auf ihrer Erfahrung, ihrer Kenntnis der einschlägigen Literatur und der Diskussion mit ihren Fachkollegen.

\subsection{Aktualisierung}

Eine Überarbeitung der Guidelines ist zwei Jahre nach der Publikation vorgesehen.

\subsection{Implementierung}

Die Implementierung der Guideline erfolgt über Publikationen in den Printmedien, Verbreitung durch Internet, Aufnahme durch die Akademie für Fortbildung der Schweizerischen Gesellschaft für Gynäkologie und Geburtshilfe (SGGG) und Einsatz in den Weiter- und Fortbildungsveranstaltungen der SGGG. Jedes Mitglied der SGGG erhält ein Exemplar zugestellt.

\section{Zusammenfassung der Empfehlungen}

Diese Empfehlungen beziehen sich auf Patientinnen mit symptomatischen oder asymptomatischen Adnextumoren.

1. Die Abklärung beginnt mit der Anamnese.

2. Das Ziel der Untersuchung ist die Unterscheidung zwischen gutartigem und bösartigem Adnexbefund.

3. Die Sonographie ist die apparative Abklärungsmethode der Wahl. Sie erlaubt, die Grösse, die Morphologie und den uni- oder multilokulären Charakter des Adnexbefundes sowie das Vorhandensein von Aszites zu bestimmen.

4. Die Verwendung morphologischer Ultraschallkriterien erlaubt, einen Score zu erstellen (z.B. Sassone oder Mainz), der die Evaluation eines Adnexbefundes ermöglicht.

5. Die Dopplersonographie erlaubt die Erkennung von rasch wachsenden Adnexbefunden. Aufgrund der Spezifität ist sie nur in der Postmenopause anwendbar.

6. In der Prämenopause bestimmt man bei einer Amenorrhoe das ß-HCG im Urin bzw. im Serum.

7. Der CA-125-Serumwert darf nicht als alleiniger Früherkennungstest verwendet werden.

8. Ein gutartiger Adnexbefund sollte nach 6-9 Wochen sonographisch nachkontrolliert werden (bei der prämenopausalen Frau in der ersten Zyklusphase).

9. Ein verdächtiger Adnexbefund (wahrscheinlich gutartig oder wahrscheinlich bösartig) muss mittels Laparoskopie weiter abgeklärt werden.

10. Ein gutartiger oder wahrscheinlich gutartiger Adnexbefund wird in der Prämenopause durch Befundausschälung und in der Postmenopause durch eine Adnexektomie behandelt. In der Postmenopause kann eine beidseitige Adnexektomie vorgeschlagen werden. 
11. Bei einem möglicherweise bösartigen Adnexbefund soll sowohl in der Prä- wie in der Postmenopause die Adnexektomie erfolgen.

12. Die Entfernung eines Adnexbefundes während einer Laparoskopie muss immer in einem Bergebeutel erfolgen.

13. Ein bösartiger Adnexbefund bedarf einer medianen Laparotomie.

14. Die alleinige Zystenpunktion und die alleinige endoskopische Inspektion des Zystenbalges gilt nicht als adäquate Therapie. Das Morcellement eines Adnexbefundes soll nicht durchgeführt werden.

15. Die Zeitspanne zwischen der Ruptur eines bösartigen Ovarialbefundes im Stadium I bis zur definitiven Laparotomie sollte 8-14 Tage nicht überschreiten.

\section{Abklärungen und weiteres Vorgehen}

\subsection{Die Abklärung eines Adnexbefundes beginnt mit der Anamnese}

Die Kenntnis des Zeitpunktes der letzten Menstruation ist essentiell. Es ist wichtig zu wissen, ob eine Patientin prä- oder postmenopausal ist, ob ihr Zyklus regelmässig ist und ob sie schwanger ist.

Das Risiko eines Malignoms steigt mit dem Alter. Die meisten Frauen, die einen malignen Ovarialtumor entwickeln, haben keine erkennbaren Risikofaktoren $\varepsilon 3$.

Im weiteren sind die Symptome von Interesse. Bestehen Schmerzen, kam es zu einer Zunahme des Abdomenumfangs, zu Appetitverlust, zu Obstipation oder zu Gewichtsverlust? Ein Adnextumor ist häufig asymptomatisch [5] $\varepsilon 3$.

\subsection{Das Ziel der klinischen Untersuchung ist die Unterscheidung zwischen gutartigem und bösartigem Adnexbefund}

Die klinische Untersuchung besteht aus der Palpation des Abdomens sowie aus der bimanuellen rektovaginalen Untersuchung. Dadurch soll festgestellt werden, ob ein Adnexbefund einseitig oder beidseitig palpabel ist, ob er mobil ist und wie seine Oberfläche und seine Konsistenz beschaffen sind [5]. Allerdings werden Befunde von 4 bis $6 \mathrm{~cm}$, respektive von 6 bis $8 \mathrm{~cm}$, nur in 33\% bzw. 75\% durch Palpation erkannt [6] \&3. Die Sensitivität der bimanuellen rektovaginalen Palpation wird auf 15 bis 36\% geschätzt [7] $\varepsilon 3$.
3.3 Die Sonographie ist die apparative Abklärungsmethode der Wahl. Sie erlaubt, die Grösse, die Morphologie und den unioder multilokulären Charakter des Adnexbefundes sowie das Vorhandensein von Aszites zu bestimmen

Zur Abklärung eines Adnextumors ist weder eine Computertomographie (CT) noch eine Magnetresonanztomographie (MRI) notwendig.

\section{Grösse}

Mit zunehmender Grösse eines Adnexbefundes steigt das Risiko für Malignität. Es beträgt $1,9-13,9 \%$ bei einem Adnexbefund von $5 \mathrm{~cm}$ Durchmesser, $7-35,6 \%$ bei 5-10 cm Durchmesser und $12,5-71,8 \%$ bei einem Tumor von über $10 \mathrm{~cm}$ Durchmesser [8-10] $\varepsilon 3$.

\section{Lokularität und Septen}

Ein multilokulärer zystischer Adnexbefund ist definiert durch das Vorhandensein von mehreren Kompartimenten, welche durch Septen abgegrenzt sind. Es kann schwierig sein, einen mehrkammerigen zystischen Adnexbefund von einem polyzystischen Ovar zu unterscheiden. Ein multilokulärer zystischer Adnexbefund hat ein deutlich höheres Malignitätsrisiko. Dieses Risiko ist bei einem unilokulären zystischen Adnexbefund $0-6,1 \%$ und bei einem multilokulären zystischen Adnexbefund 18-37\% [8-14] $\varepsilon 3$.

\section{Echogenität}

Die Echogenität des Adnexbefundes wird durch dessen Inhalt bestimmt. Aufgrund der Echogenität kann unterschieden werden, ob ein zystischer Adnexbefund nur Flüssigkeit oder auch solide Strukturen beinhaltet. Ein seröser, zystischer Adnexbefund ist echofrei. Ein muzinöser Inhalt hat einen gräulichen Aspekt. Demgegenüber erscheinen solide Strukturen (z. B. ein Zahn) hyperechogen und können einen Schallschatten haben [11]. Dieses Phänomen sieht man bei $82 \%$ der Teratome und bei $28 \%$ der Borderline- und malignen Tumore [15] $\varepsilon 3$.

\section{Wanddicke und Septen}

Ein gutartiger zystischer Adnexbefund ist dünnwandig und glatt. Maligne Tumoren haben dickere und unregelmässigere Wände und Septen. Definitionsgemäss gilt ein Septum von mehr als $3 \mathrm{~mm}$ als dick. Diese Kriterien sind nicht immer Zeichen einer Malignität, da auch die Wände eines Teratoms und eines Endometrioms dick sein können [15]. $\varepsilon 3$ 
Papilläre Auflagerungen, intrazystische Vegetationen

Intrazystische Vegetationen (papilläre Auflagerungen) bestehen aus einem unterschiedlich dichten Stroma, bedeckt von Epithel. Papilläre Auflagerungen sind mit einem Risiko für Malignität von 40,5 bis $83,7 \%$ behaftet. Je mehr papilläre Auflagerungen vorhanden sind, desto grösser ist das Malignitätsrisiko [11]. $\varepsilon 3$

Alle Studien zusammengefasst hat die transvaginale Ultraschalluntersuchung eine Sensitivität bezüglich Malignomen von 69 bis 98\%, eine Spezifität von 52 bis 91,2\%, einen positiven prädiktiven Wert von 37 bis $60 \%$ und einen negativen prädiktiven Wert von 92,6 bis $100 \%$ [15-27]. \&3

\subsection{Die Verwendung morphologischer Ultraschallkriterien erlaubt einen Score zu erstellen (z. B. Sassone oder Mainz), der die Evaluation eines Adnexbefundes ermöglicht}

Die Grösse eines Adnexbefundes, seine Struktur, seine Echogenität, die Dicke seiner Wand und der Nachweis von Septen, das Vorhandensein von Vegetationen und die Präsenz von Aszites sind entscheidende Kriterien für die Scoreberechnung [15-21, 27]. Ein einkammriger, zystischer Befund mit feiner, glatter Wand hat eine niedrige Punktzahl, dagegen wird ein multilokulärer Befund mit dicker Wand und echogenem Inhalt mit einer hohen Punktzahl (Tab. 2 und 3) bewertet. Ein Adnexbefund kann bösartig sein, sofern $\geq 9$ im Sassonescore [16] erreicht werden (Tab. 2). Verwendet man den Mainzer-Score, so sprechen $\geq 9$ in der Prämenopause und $\geq 10$ in der Postmenopause für einen bösartigen Befund (Tab. 3) [21, 27]. \&3

Die Punktzahlen für die einzelnen empfohlenen Scores sind nicht immer reproduzierbar. Es bestehen nur wenige, prospektive Untersuchungen mit prospektiver Evaluation bzw. Validierung der erwähnten Scores [22].

Man kann sich in der Regel nicht damit begnügen, alle morphologischen Charakteristiken $\mathrm{zu}$ summieren, um $\mathrm{zu}$ entscheiden, ob eine Intervention notwendig ist, da sonst eine $\mathrm{zu}$ hohe falsch-positive Rate entsteht. Um maligne Prozesse nicht zu verpassen, darf man sich auch nicht durch einen Score limitieren lassen [11].

Tabelle 1

Spezifität, Sensitivität und prädiktiver Wert der Transvaginalsonographie.

\begin{tabular}{|c|c|c|c|c|c|c|c|c|c|c|}
\hline Nr. & Jahr & Studien & $\mathbf{n}$ & Aussage & Spezifität & Sensitivität & $\begin{array}{l}\text { Negativ } \\
\text { prädiktiver } \\
\text { Wert }\end{array}$ & $\begin{array}{l}\text { Positiv } \\
\text { prädiktiver } \\
\text { Wert }\end{array}$ & Evidenz & Validität \\
\hline 8 & 1990 & Granberg et al. & 180 & $\begin{array}{l}\text { Erhöhte Wahrscheinlichkeit für } \\
\text { Malignität bei grossen Tumoren } \\
\text { und papillären Strukturen }\end{array}$ & 52 & 91 & - & 52 & III & - \\
\hline 16 & 1991 & Sassone et al. & 143 & $\begin{array}{l}\text { Score, welcher die Unterschei- } \\
\text { dung zwischen gutartigen und } \\
\text { bösartigen Tumoren erleichtert }\end{array}$ & 83 & 100 & 100 & 37 & III & - \\
\hline 37 & 1992 & Weiner et al. & 53 & $\begin{array}{l}\text { Diagnostikverbesserung } \\
\text { dank Dopplersonographie }\end{array}$ & 69 & 94 & 96 & 59 & III & - \\
\hline 38 & 1993 & Timor-Tritsch et al. & 115 & $\begin{array}{l}\text { Diagnostikverbesserung } \\
\text { dank Dopplersonographie }\end{array}$ & 87 & 94 & 99 & 60 & III & - \\
\hline 26 & 1994 & Bromley et al. & 33 & $\begin{array}{l}\text { Keine Diagnostikverbesserung } \\
\text { durch Dopplersonographie }\end{array}$ & 52 & 91 & - & 52 & III & - \\
\hline 29 & 1994 & Jain & 50 & $\begin{array}{l}\text { Diagnostikverbesserung } \\
\text { dank Dopplersonographie }\end{array}$ & 95 & 90 & - & - & III & 94 \\
\hline 20 & 1995 & Hata et al. & 102 & $\begin{array}{l}\text { Diagnostikverbesserung } \\
\text { dank Dopplersonographie }\end{array}$ & 69 & 87 & 93 & 54 & III & 75 \\
\hline 41 & 1995 & Stein et al. & 170 & $\begin{array}{l}\text { Keine Diagnostikverbesserung } \\
\text { durch Dopplersonographie }\end{array}$ & 62 & 98 & 99 & 50 & III & - \\
\hline 42 & 1995 & Zanetta et al. & 150 & $\begin{array}{l}\text { Diagnostikverbesserung } \\
\text { dank Dopplersonographie }\end{array}$ & 74 & 89 & - & - & III & 82 \\
\hline 25 & 1996 & Leeners et al. & 109 & $\begin{array}{l}\text { Diagnostikverbesserung } \\
\text { dank Dopplersonographie }\end{array}$ & 85 & 70 & - & - & III & - \\
\hline 43 & 1996 & Prömpeler et al. & 212 & $\begin{array}{l}\text { Diagnostikverbesserung } \\
\text { dank Dopplersonographie }\end{array}$ & 63 & 91 & - & - & III & 77 \\
\hline 27 & 1999 & Weber et al. & 195 & $\begin{array}{l}\text { Score, welcher die Unterschei- } \\
\text { dung zwischen gutartigen und } \\
\text { bösartigen Tumoren erleichtert }\end{array}$ & 91 & 97 & 97 & 91 & III & - \\
\hline
\end{tabular}


Tabelle 2

Sassone-Score [16].

\begin{tabular}{lllll} 
& Struktur der Innenwand & Wanddicke & Septen & Echogenität \\
\hline 1 & glatt & Dünn $\leq 3 \mathrm{~mm}$ & keine & keine \\
\hline 2 & unregelmässig $\leq 3 \mathrm{~mm}$ & dick $>3 \mathrm{~mm}$ & dünn $\leq 3 \mathrm{~mm}$ & gering \\
\hline 3 & papilläre Strukturen $>3 \mathrm{~mm}$ & vorwiegend solid & dick $>3 \mathrm{~mm}$ & $\begin{array}{l}\text { gering und echo- } \\
\text { genes Zentrum }\end{array}$ \\
\hline 4 & vorwiegend solid & & gemischt \\
\hline 5 & & & stark
\end{tabular}

Tabelle 3

Score nach Mainz.

\begin{tabular}{|c|c|c|c|c|}
\hline Score & 0 & 1 & 2 & Total \\
\hline Gesamtstruktur des Tumors & - & einfach & komplex & 2 \\
\hline Begrenzung des Tumors & glatt & leicht irregulär & deutlich irregulär & 2 \\
\hline Wanddicke & $<3 \mathrm{~mm}$ & $\geq 3, \leq 5 \mathrm{~mm}$ & $\begin{array}{l}>5 \mathrm{~mm} \text {, oder nicht } \\
\text { beurteilbar }\end{array}$ & 2 \\
\hline $\begin{array}{l}\text { Binnenechos im zystischen } \\
\text { Anteil }\end{array}$ & keine & homogen & inhomogen & 2 \\
\hline Septen & keine & $\leq 3 \mathrm{~mm}$ & $>3 \mathrm{~mm}$ & 2 \\
\hline $\begin{array}{l}\text { Form des komplexen oder } \\
\text { rein soliden Anteils }\end{array}$ & keine & glatt & höckerig & 2 \\
\hline $\begin{array}{l}\text { Echogenität des komplexen } \\
\text { oder rein soliden Anteils }\end{array}$ & keine & homogen & inhomogen & 2 \\
\hline Schallschatten & Echoverstärkung & teilweise & vollständig & 2 \\
\hline Aszites & keiner & wenig & mässig & 2 \\
\hline $\begin{array}{l}\text { Lebermetastasen / } \\
\text { Peritonealkarzinose }\end{array}$ & $\begin{array}{l}\text { nicht } \\
\text { nachweisbar }\end{array}$ & $\begin{array}{l}\text { nicht schlüssig } \\
\text { beurteilbar }\end{array}$ & darstellbar & 2 \\
\hline
\end{tabular}

3.5 Die Dopplersonographie erlaubt die Erkennung von rasch wachsenden Adnexbefunden. Aufgrund der Spezifität ist sie nur in der Postmenopause anwendbar

Der Farbdoppler erlaubt, die Vaskularisation von Adnexbefunden zu analysieren. Die angiogene Aktivität eines Tumors korreliert mit dem Wachstum der Geschwulst. Die Gefässe und die Shunts der Tumoren mit schnellem Wachstum haben eine unterschiedliche Beschaffenheit der Tunica muscularis. In der Diastole resultiert daraus nicht nur ein geringerer Gefässwiderstand, sondern auch eine geringere Pulsatilität. Daraus resultierten eine Verringerung des Resistance Index und des Pulsility Index und eine Zunahme der Velocity [28-31]. Man beobachtet einen fortlaufenden diastolischen Fluss. Dieses Phänomen sieht man in $68-98 \%$ der bösartigen Tumoren, in 35-99\% der gutartigen Tumoren wie auch beim Gelbkörper [32-37, 40]. \&3

Nicht alle bösartigen Tumoren zeigen eine Zunahme des Flows in Gefässen, denn das Lumen der Gefässe kann karzinombedingt von Thrombosen obstruiert werden [11].
Der negative prädiktive Wert des Farbdopplers liegt im Bereich von 89,6 bis 99,8\%, während sein positiver prädiktiver Wert bei $28,0-98,2 \%$ liegt [20, 26, 34-47]. In der Postmenopause kann der Farbdoppler eher einen bösartigen von einem gutartigen Tumor unterscheiden, während in der Prämenopause diese Unterscheidung viel unsicherer ist (Tab. 4) [3, 11].

Bei Tumoren mit einem Durchmesser von über $10 \mathrm{~cm}$ ist der Einsatz des Farbdopplers für die Entscheidung, ob ein Eingriff gemacht werden soll, nicht nötig, da die Grösse allein schon Grund genug für den Eingriff ist. [11]. $\varepsilon 3$

\subsection{In der Prämenopause bestimmt man bei einer Amenorrhoe das ß-HCG im Urin bzw. im Serum}

Ein Adnexbefund in Kombination mit einem positiven Serum-ß-HCG-Wert und einer leeren Gebärmutterhöhle lässt die Existenz einer extrauterinen Schwangerschaft vermuten [5]. E3

3.7 Der CA-125-Serumwert darf nicht als alleiniger Früherkennungstest verwendet werden Die Sensibilität dieses Tumormarkers liegt im Stadium I bei nur 50\%, während sie bei den Stadien II-IV bei 90\% liegt (Tab. 5) [48-56]. $\varepsilon 3$

Das CA-125 kann auch bei den folgenden benignen Veränderungen erhöht sein: Menstruation, Endometriose, Myomen, Schwangerschaft, pelvinen Infektionen, Leberaffektionen, gastrointestinalen Erkrankungen und bei Perikarditis. Ausserdem kann der Tumormarker auch bei anderen Krebserkrankungen ansteigen, wie zum Beispiel bei Brustkrebs, beim Endometriumkarzinom, bei Lungenkrebs, Lebermalignomen, Harnblasenkrebs, Pankreaskrebs und bei NonHodgkin-Lymphomen [52, 57]. Dies erklärt, warum bei $98 \%$ der Frauen, bei denen ein erhöhtes CA-125 als Zufall gefunden wird, kein Ovarialkarzinom vorliegt [58]. \&3

Weitere Tumormarker wie $\alpha$-FP, CA-72.4 und OVX-1 u.a. sind ebenfalls ungeeignet für den alleinigen Einsatz in der Früherkennung des Ovarialkarzinoms.

\subsection{Ein gutartiger Adnexbefund sollte nach} 6-9 Wochen sonographisch nachkontrolliert werden (bei der prämenopausalen Frau in der ersten Zyklusphase)

In der Prämenopause verschwinden 89\% aller funktionellen Adnexbefunde wieder [22]. In der Postmenopause kommt es in $37-40 \%$ aller unilokulären, zystischen Adnexbefunde, die $5-10 \mathrm{~cm}$ messen, in weniger als zwei Monaten zu einer Verkleinerung [23, 24]. 55\% der komplizierten zystischen Befunde, die entweder multilokulär 
und/oder solid sind und nicht $10 \mathrm{~cm}$ an Grösse übersteigen, können ebenfalls verschwinden [24]. ع3 Sofern mit der Patientin eine Ultraschallkontrolle vereinbart wird, soll diese nach 6-9 Wochen durchgeführt werden [22]. Bei den prämenopausalen Frauen wird diese Untersuchung in der ersten Zyklusphase empfohlen (Abb. 1 und 2). Die Persistenz eines komplexen, zystischen Befundes erhöht das Malignitätsrisiko, unabhängig von allen morphologischen Scores [22].

\subsection{Ein verdächtiger Adnexbefund (wahr- scheinlich gutartig oder wahrscheinlich bösartig) muss mittels Laparoskopie weiter abgeklärt werden}

Patientinnen mit Adnexbefunden, die ein kleines Risiko haben, bösartig zu sein, können gut laparoskopisch behandelt werden [59, 60]. Die Vorteile der Laparoskopie sind: kürzere Hospitalisation [61-63], weniger postoperative Schmerzen [63, 64], weniger Adhäsionen [65] und tiefere Kosten [66]. \&3 Trotz der Vorteile der Laparoskopie soll man nie die Prinzipien der Krebschirurgie verletzen [59]. Das Einverständnis der Patientin für eine Laparotomie ist aus diesen Gründen immer präoperativ einzuholen.
$\mathrm{Zu}$ Beginn der Laparoskopie inspiziert man die ganze Peritonealhöhle. Entdeckt man einen bösartigen Tumor oder hat man den starken Verdacht, einen solchen entdeckt zu haben, ist die direkte Laparotomie gerechtfertigt. Man führt eine Peritoneallavage durch oder entnimmt allfälligen Aszites zur zytologischen Diagnostik. Ein gutartiges Aussehen eines Adnexbefundes ist kein sicherer Beweis für eine benigne Histologie. Der kontralaterale Eierstock muss stets gut inspiziert und allenfalls biopsiert werden, ebenso ist eine Douglas-Zytologie zu entnehmen.

Es wird empfohlen, am Ende des Eingriffs die ganze Peritonealhöhle ausgiebig zu spülen [60].

\subsection{Ein gutartiger oder wahrscheinlich gutartiger Adnexbefund wird in der Prä- menopause durch Befundausschälung und in der Postmenopause durch eine Adnex- ektomie behandelt. In der Postmenopause kann eine beidseitige Adnexektomie vorgeschlagen werden}

In der Prämenopause kann bei Auffinden eines solchen Befundes die Operation limitiert und nur eine Befundausschälung durchgeführt werden. Im Idealfall entnimmt man den Befund

Tabelle 4

Spezifität, Sensitivität und prädiktiver Wert der Dopplersonographie.

\begin{tabular}{|c|c|c|c|c|c|c|c|c|c|c|}
\hline Nr. & Jahr & Studien & $\mathbf{n}$ & Variablen & Spezifität & Sensitivität & $\begin{array}{l}\text { Negativ } \\
\text { prädiktiver Wert }\end{array}$ & $\begin{array}{l}\text { Positiv } \\
\text { prädiktiver Wert }\end{array}$ & Evidenz & Validität \\
\hline 34 & 1990 & Kurjak et al. & 147 & $\mathrm{RI}<0,4$ & 99 & 100 & - & - & III & 99 \\
\hline 35 & 1990 & Kurjak et al. & 151 & RI 0,5 & 100 & 100 & 100 & 100 & III & 100 \\
\hline 36 & 1992 & Kurjak et al. & 83 & RI 0,41 & 65 & 93 & 95 & 59 & III & - \\
\hline 37 & 1992 & Weiner et al. & 53 & PI 1,0 & 97 & 94 & 97 & 94 & III & - \\
\hline \multirow[t]{2}{*}{38} & 1993 & Timor-Tritsch et al. & 115 & RI 0,46 & 99 & 94 & 99 & 94 & III & - \\
\hline & & & 115 & PI 0,62 & 97 & 88 & 97 & 88 & & - \\
\hline \multirow[t]{2}{*}{39} & 1994 & Wu et al. & 222 & RI 0,4 & 94 & 67 & - & - & III & - \\
\hline & & & 222 & RI 0,5 & 90 & 75 & - & - & & - \\
\hline 26 & 1994 & Bromley et al. & 33 & RI 0,6 & 81 & 66 & 67 & - & III & 66 \\
\hline \multirow[t]{2}{*}{40} & 1995 & Carter et al. & 123 & $\mathrm{PI}<1,0$ & 79 & 57 & 89 & 30 & III & 75 \\
\hline & & & 123 & RI 0,6 & 77 & 43 & 86 & 55 & & 70 \\
\hline \multirow[t]{2}{*}{20} & 1995 & Hata et al. & 102 & $\mathrm{RI}<0,72$ & 68 & 93 & 96 & 81 & III & 76 \\
\hline & & & 102 & $\mathrm{PI}>16 \mathrm{~cm} /$ Sekunde & 92 & 83 & 93 & 46 & & 89 \\
\hline \multirow[t]{2}{*}{41} & 1995 & Stein et al. & 170 & PI 1,0 & 66 & 67 & 83 & 50 & III & - \\
\hline & & & 170 & RI 0,5 & 90 & 24 & 73 & - & & - \\
\hline \multirow[t]{2}{*}{42} & 1995 & Zanetta et al. & 150 & $\mathrm{PI}<1,0$ & 73 & 93 & - & - & III & 83 \\
\hline & & & 150 & $\mathrm{RI}<0,56$ & 80 & 85 & - & - & & 82 \\
\hline \multirow[t]{2}{*}{43} & 1996 & Prömpeler et al. & 212 & $\begin{array}{l}\text { RI } 0,5 \\
\text { (prämenopausal) }\end{array}$ & 59 & 86 & - & - & III & 66 \\
\hline & & & 212 & $\begin{array}{l}\text { RI } 0,5 \\
\text { (postmenopausal) }\end{array}$ & 55 & 79 & - & - & & 69 \\
\hline \multirow[t]{2}{*}{44} & 1998 & Emoto et al. & 143 & $\mathrm{RI}<0,51$ & 77 & 91 & 98 & 45 & III & 75 \\
\hline & & & 143 & $\mathrm{PI}<0,86$ & 72 & 100 & 100 & 42 & & 75 \\
\hline
\end{tabular}


Tabelle 5

Spezifität, Sensitivität und prädiktiver Wert des CA-125.

\begin{tabular}{|c|c|c|c|c|c|c|c|c|c|c|}
\hline Nr. & Jahr & Studien & $\mathbf{n}$ & Aussage & Spezifität & Sensitivität & $\begin{array}{l}\text { Negativ } \\
\text { prädiktiver } \\
\text { Wert }\end{array}$ & $\begin{array}{l}\text { Positiv } \\
\text { prädiktiver } \\
\text { Wert }\end{array}$ & Evidenz & Validität \\
\hline 50 & 1988 & Malkasian et al. & 158 & $\begin{array}{l}\text { Spezifität und Sensitivität sind besser } \\
\text { in der Postmenopause }\end{array}$ & 80 & 82 & 89 & 70 & III & 81 \\
\hline 51 & 1992 & Inoue et al. & 65 & $\begin{array}{l}\text { Die alleinige Bestimmung des CA-125 } \\
\text { ungenügend }\end{array}$ & - & 38 & - & - & III & - \\
\hline 52 & 1992 & Gadducci et al. & 344 & $\begin{array}{l}\text { Die Sensitivität ist besser in der Post- } \\
\text { menopause }\end{array}$ & 76 & 87 & 99 & 60 & III & 69 \\
\hline 53 & 1994 & Maggino et al. & 388 & $\begin{array}{l}\text { Diagnostikverbesserung dank } \\
\text { Dopplersonographie }\end{array}$ & 96 & 92 & - & - & III & 94 \\
\hline 54 & 1994 & Schutter et al. & 228 & $\begin{array}{l}\text { Diagnostikverbesserung dank Klinik } \\
\text { und Dopplersonographie }\end{array}$ & 80 & 72 & - & - & III & - \\
\hline 55 & 1996 & Gadducci et al. & 124 & $\begin{array}{l}\text { Erhöhtes Risiko für Malignität bei } \\
\text { erhöhtem CA-125 \& D-Dimer }\end{array}$ & 94 & 77 & 82 & 92 & III & - \\
\hline 56 & 1996 & Dorum et al. & 180 & $\begin{array}{l}\text { Die alleinige Bestimmung des CA-125 } \\
\text { ist ungenügend }\end{array}$ & 97 & 44 & 97 & 44 & III & - \\
\hline
\end{tabular}

intakt ohne Eröffnung und Entleerung des Inhalts in die Peritonealhöhle. 15-20\% aller sogenannten funktionellen Zysten zeigen histologisch doch organische Veränderungen [67]. $\varepsilon 3$

Wenn der Befund mehr als 8-10 cm misst und wenn die Wand sehr dünn ist, besteht die Gefahr einer Ruptur. Um die Folgen einer Ruptur zu verhindern, plaziert man den Befund zur Entfernung in einen Bergesack.

In der Peri- und Postmenopause sollte man eine uni- bzw. je nach Situation und Einver-

Abbildung 1

Adnexbefund in der Prämenopause.

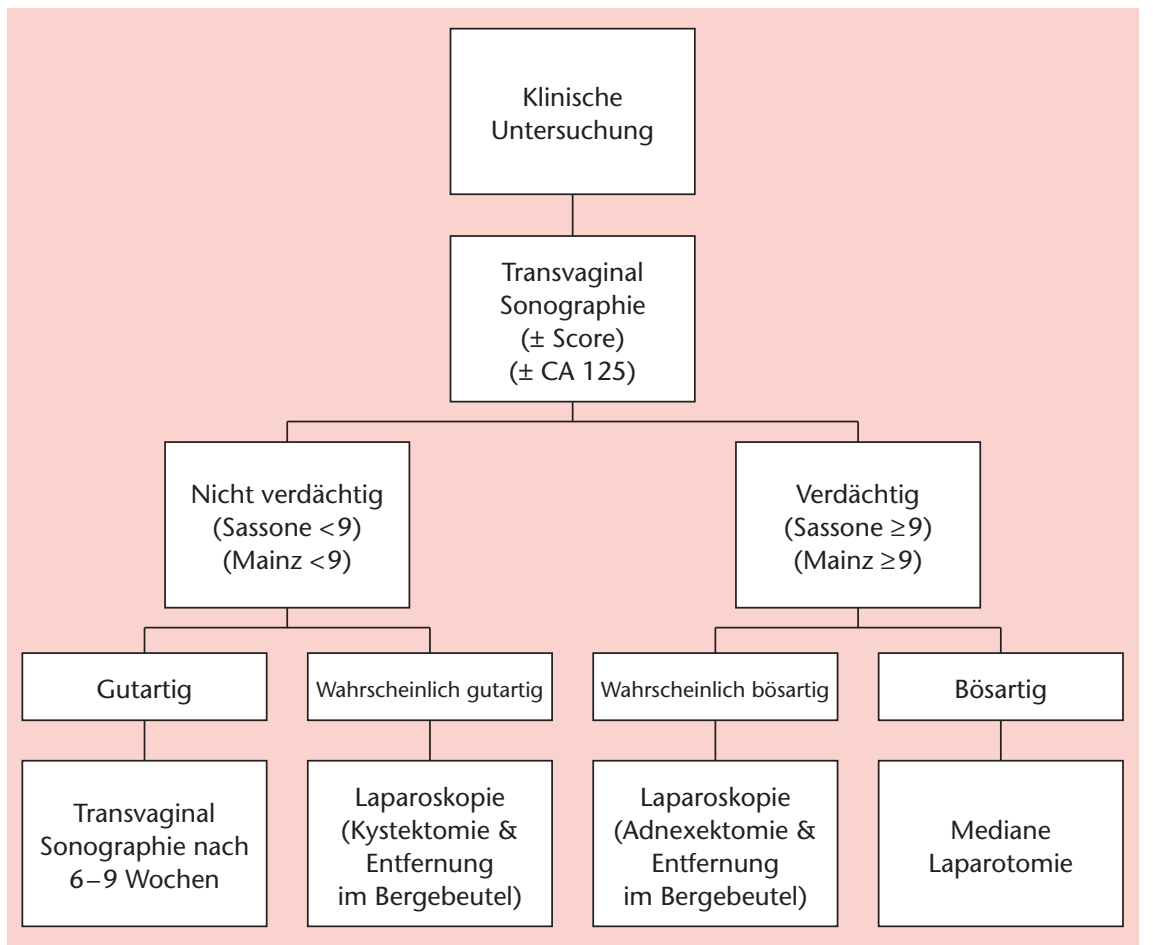

ständnis der Patientin eine bilaterale Adnexektomie durchführen, ohne den Befund zu rupturieren [68]. Der Uterus kann erhalten bleiben.

Bei Zweifel hinsichtlich der Gutartigkeit des entfernten Adnexbefundes ist eine Schnelldiagnose bzw. Schnellschnittdiagnose wertvoll und anzustreben [69].

\subsection{Bei einem möglicherweise bösartigen Adnexbefund soll, sowohl in der Prä- wie in der Postmenopause, die Adnexektomie erfolgen}

Die Ausschälung eines Adnexbefundes, der als wahrscheinlich bösartig beurteilt wird, birgt das Risiko der Zellverschleppung in die Abdominalhöhle und ist deshalb in der Prä- und Postmenopause zu vermeiden.

Die Möglichkeit, während der Laparoskopie eine Schnellschnittuntersuchung durchzuführen, erlaubt, falls nötig, ohne Verzögerung die Durchführung einer Laparotomie [69-70].

\subsection{Die Entfernung eines Adnexbefundes während einer Laparoskopie muss immer in einem Bergebeutel erfolgen}

Der Adnexbefund muss in einen Bergebeutel plaziert werden [60], anschliessend wird der Bergebeutel verschlossen und der Befund im Bergebeutel geborgen. Solange sich der Bergebeutel in der Abdominalhöhle befindet, sollte unbedingt die Perforation verhindert werden. Ebenso sollte jede mögliche, peritoneale Kontamination von Tumorzellen verhindert werden. Laborergebnisse zeigen, dass Kohlenstoffdioxyd $\left(\mathrm{CO}_{2}\right)$ das Wachstum von Tumorzellen im Peritoneum stimulieren kann [71]. \&3 
Sofern eine bilaterale Adnexektomie durchgeführt wird, können auch zwei Eierstöcke in den gleichen Sack plaziert werden. Bei makroskopisch gleichartigen Befunden ist die Seitenkennzeichnung notwendig.

\subsection{Ein bösartiger Adnexbefund bedarf einer medianen Laparotomie}

Eine mediane Laparotomie unterhalb bzw. je nach Befund oberhalb des Nabels ist notwendig, sofern man die Diagnose bereits präoperativ gestellt hat und/oder wenn der bösartige Befund während einer Laparoskopie diagnostiziert wurde. In dieser Situation ist mit Adhäsionen und Tumorauflagerungen auf den Nachbarorganen zu rechnen. $\mathcal{E} 4$

Die Intervention beinhaltet eine Peritoneallavage, eine bilaterale Adnexektomie, eine Hysterektomie, eine infrakolische Omentektomie, Peritonealbiopsien, eine pelvine und paraaortale Lymphadenektomie (besonders bei Patientinnen in Frühstadien) und eine Blinddarmentfernung [69].

\subsection{Die alleinige Zystenpunktion und} die alleinige endoskopische Inspektion des Zystenbalges gilt nicht als adäquate Therapie. Das Morcellement eines Adnexbefundes soll nicht durchgeführt werden Die Punktion eines Adnexbefundes führt $\mathrm{zu}$ einem erhöhten Rezidivrisiko. $\varepsilon 4$ Die Aspiration oder die Fenestrierung bzw. die partielle Exzision

Abbildung 2

Adnexbefund in der Postmenopause.

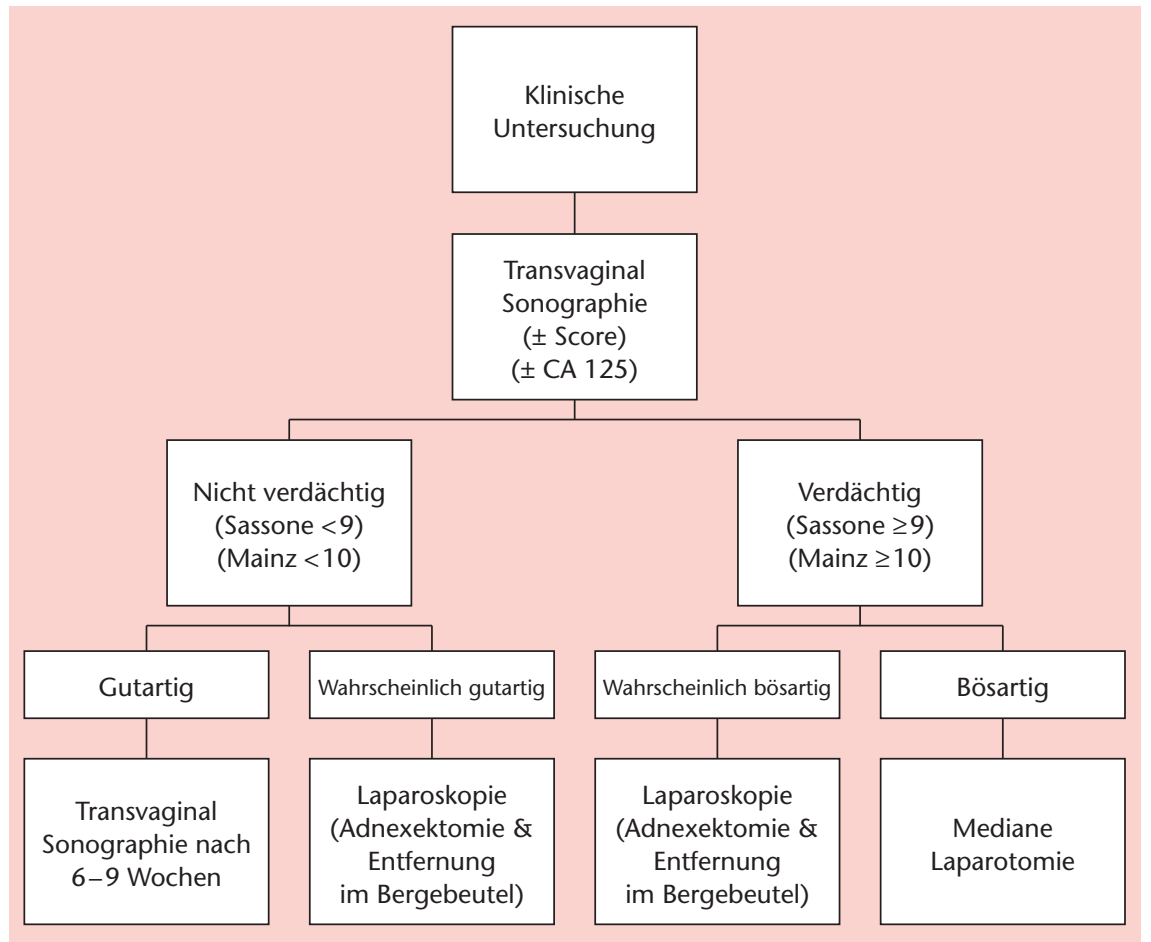

sind nur bei persistierenden Follikelzysten und bei Endometriomen im Rahmen weiterer Therapien sinnvoll [60].

Die Ausschälung und/oder das Morcellement eines wahrscheinlich bösartigen oder bösartigen Befundes kann eine Aussaat von Tumorzellen in der Abdominalhöhle zur Folge haben. Die Prognose kann verschlechtert werden [69, 72], wenn es sich um ein Karzinom oder ein Teratom (chemische Peritonitis) handelt [73]. \&3

Die intraoperative Ruptur eines bösartigen Adnexbefundes kann sowohl während einer Laparotomie wie auch während einer Laparoskopie erfolgen. Im Falle eines Ovarialkarzinoms Stadium I erhöht die Ruptur das Rückfallrisiko, während die direkten Folgen auf das Gesamtüberleben ungewiss sind [74-78].

\subsection{Die Zeitspanne zwischen Ruptur eines bösartigen Ovarialbefundes im Stadium I bis zur definitiven Laparotomie sollte nicht 8-14 Tage überschreiten}

Die Zeitspanne zwischen Ruptur - oder Dauer von der unvollständigen laparoskopischen Entfernung eines Ovarialmalignoms - bis zur definitiven Laparotomie ist ein wichtiger Prognosefaktor. Dies wurde von Kindermann et al. [69], Lehner et al. [72] und von Maiman et al. [79] gezeigt. Ein solcher Tumor kann in der Folge Progredienz zeigen, wenn die Frist von 8 Tagen [69] bzw. von 17 Tagen [72] bzw. von 4 Wochen [79] überschritten wird. $\varepsilon 3$ Es gilt zu betonen, dass über die Langzeitfolgen bei Patientinnen mit laparoskopisch anoperierten Malignomen noch wenig bekannt ist [69]. Die Arbeitsgruppe empfiehlt deshalb, sich an die Frist von max. 8 bis 14 Tagen bis zur Reoperation zu halten.

\section{Literatur}

1 Krebs in der Schweiz: Fakten, Kommentare. Bern: Schweizerische Krebsliga;1998.

2 Prömpeler HJ, Madjar H, Sauerbrei W, Lattermann, Pfleiderer A. Diagnostic formula for the differentiation of adnexal tumors by transvaginal sonography. Obstet Gynecol 1997;89:428-33.

3 Kuhn W, Ohmers R. Frühdiagnostik und Management von Ovarientumoren. In: von Künzel W, Kirschbaum M (Hrsg.). Fortbildungskongress für Ärzte der Frauenheilkunde und Geburtshilfe. Heidelberg: Springer; 1993. S. 271-6.

4 Clinical practice guidelines for the care and treatment of breast cancer: a Canadian consensus document. CMAJ 1998;158 (3 Suppl).

5 Beck L, Berg D, Leidenberger F, et al. Die Abklärung eines Adnexbefundes. Gynäkologe 1999;32:467-84. 
6 Pinotti JA, De Franzin CM, Marussi EF, Zeferino LC. Evolution of cystic and adnexal tumors identified by echography. Int J Gynaecol Obstet 1988; 26:109-14.

7 Padilla LA, Radosevich DM, Milad MP. Accuracy of the pelvic examination in detecting adnexal masses. Obstet Gynecol 2000;96:593-8.

8 Granberg S, Wikland M, Janson I. Macroscopic characterisation of ovarian tumors and the relation to the histological diagnosis: criteria to be used for ultrasound evaluation. Gynecol Oncol 1989;35:139-44.

9 Granberg S, Norstrom A, Wikland M. Tumors in the lower pelvis as imaged by vaginal sonography. Gynecol Oncol 1990;37:224-9.

10 Luxman D, Bergman A, Sagi J, David MP. The postmenopausal adnexal mass: correlation between ultrasonic and pathologic findings. Obstet Gynecol 1991;77:726-8.

11 Tailor A, Hacket E, Bourne T. Ultrasonography of the ovary. In: Anderson JC (ed.). Gynecologic Imaging. London: Churchill Livingstone; 1999. p. 319-43.

12 Valentin L, Sladkedevicius P, Marsal K. Limited contribution of Doppler velocimetry to the differential diagnosis of extrauterine pelvic tumours. Obstet Gynecol 1994;83:425-33.

13 Hermann U, Locher GW, Goldhirsch A. Sonographic patterns of ovarian tumors: prediction of malignancy. Obstet Gynecol 1987;69:777-81.

14 Bourne TH, Campbell S, Reynolds KM, Whitehead MI, Hampson J, Royston P, et al. Screening for early familial cancer with transvaginal ultrasonography and colour blood flow imaging. Br Med J 1993;306:1025-9.

15 Lerner JP, Timor-Tritsch JE, Federmann A, Abramovich G. Transvaginal ultrasonographic characterisation of ovarian masses with an improved, weighted scoring system. Am J Obstet Gynecol 1994;170:81-5.

16 Sassone AM, Timor-Tritsch IE, Artner A, Westhoff C, Warren WB. Transvaginal sonographic characterisation of ovarian disease: evaluation of a new scoring system to predict ovarian malignancy. Obstet Gynecol 1991;78:70-5.

17 Kurjak A, Predanic M. New scoring system for prediction of ovarian malignancy based on transvaginal color Doppler. J Ultrasound Med 1992; 11:631-8.

18 Timor-Tritsch JE, Lerner JP, Monteguado A, Santos R. Transvaginal ultrasonographic characterisation of ovarian masses by means of color flow - direct doppler - measurements and morphologic scoring system. Am J Obstet Gynecol 1993;168:909-13

19 DePriest PD, Shenson D, Fried A, Hunter JE, Andrews SJ, Gallion HH, et al. A morphology index based on sonographic findings in ovarian cancer. Gynecol Oncol 1993;51:7-11.

20 Hata K, Hata T, Kitao M. Intratumoral peak systolic velocity as a new possible predictor for detection of adnexal malignancy. Am J Obstet Gynecol 1995;172:1496-500.
21 Merz E, Weber G, Bahlmann F, Kiesslich R. Ein neues sonomorphologisches Score-System (Mainzer-Score) zur Beurteilung von Ovarialtumoren bei der transvaginalen Sonographie. Teil I: Vergleich zwischen Score-System und Beurteilung durch erfahrenen Untersucher bei prämenopausalen Frauen. Ultraschall Med 1998; 19:99-107.

22 Osmers RGW, Osmers M, von Maydell B, Wagner B, Kuhn W. Preoperative evaluation of ovarian tumors in the premenopause by transvaginosonography. Am J Obstet Gynecol 1996; 175:428-34.

23 Kroon E, Andolf E. Diagnosis and follow-up of simple ovarian cysts by ultrasound in postmenopausal women. Obstet Gynecol 1995; 85:211-4.

24 Bailey CL, Uelan FR, Land GL, DePriest PD, Gallion HH, Kryscio RJ, van Nagell JR Jr. The malignant potential of small cystic ovarian tumors in women over 50 years of age. Gynecol Oncol 1998;69:3-7.

25 Leeners B, Schild RL, Funk S, Hauptmann S, Kemp B, Schröder W, Rath W. Colour Doppler sonography improves the preoperative diagnosis of ovarian tumours made using conventional transvaginal sonography. Eur J Obstet Gynecol 1996;64:79-85.

26 Bromley B, Goodman H, Benacerraf BR. Comparison between sonographic morphology and Doppler waveform for the diagnosis of ovarian malignancy. Obstet Gynecol 1994;83:434-7.

27 Weber G, Merz E, Bahlmann F, Leber AM. A new sonomorphologic scoring system (Mainz Score) for the assessment of ovarian tumors using transvaginal ultrasonography. Part II: A comparison between the scoring system and the assessment by an experienced sonographer in postmenopausal women. Ultraschall Med 1999;20:2-8.

28 Folkman J. How is blood vessel growth regulated in normal and neoplastic tissue? - G. H. A. Clowes Memorial Award Lecture. Cancer Res 1986;46:467-73.

29 Jain KA. Prospective evaluation of adnexal masses with endovaginal gray-scale and duplex and color Doppler US: correlation with pathologic findings. Radiology 1994;191:63-7.

30 Taylor KJM, Ramos I, Carter D, Morse IS, Snower D, Fortune K. Correlation of Doppler US tumor signals with neovascular morphologic features. Radiology 1988;166:57-62.

31 DePriest PD, Gallion HH, Pavlik EJ, Kryscio RJ, van Nagell JR. Transvaginal sonography as a screening method for the detection of early ovarian cancer. Gynecol Oncol 1997;65:408-14.

32 Folkman J. Tumor angiogenesis. Adv Cancer Res $1985 ; 43: 175-203$.

33 Bourne T. Transvaginal color Doppler in gynecology (review). Ultrasound Obstet Gynecol 1991; 1:359-73.

34 Kurjak A, Zalud I. Transvaginal colour flow imaging and ovarian cancer. Br Med J 1990;300:330. 
35 Kurjak A, Zalud I, Alfirevic Z, Jurkovic D. The assessment of abnormal pelvic blood flow by transvaginal color and pulsed Doppler. Ultrasound Med Biol 1990;16:437-42.

36 Kurjak A, Schulma H, Sosic A, Zalud I, Shalan H. Transvaginal ultrasound, color flow, and Doppler waveform of the postmenopausal adnexal mass. Obstet Gynecol 1992;80:917-21.

37 Weiner Z, Thaler I, Beck, Rottem S, Deutsch M, Brandes JM. Differentiating malignant from benign ovarian tumors with transvaginal color flow imaging. Obstet Gynecol 1992;79:159-62.

38 Timor-Tritsch LE, Lerner JP, Monteagudo A, Santos R. Transvaginal ultrasonographic characterisation of ovarian masses by means of color flow-directed Doppler measurements and a morphologic scoring system. Am J Obstet Gynecol 1993;168:909-13.

39 Wu CC, Lee CN, Chen TM, Shyu MK, Hsieh CY, Chen HY, Hsieh FJ. Incremental angiogenesis assessed by color Doppler ultrasound in the tumorgenesis of ovarian neoplasms. Cancer 1994; 73:1251-6.

40 Carter JR, Lau M, Fowler JM, Carlson JW Carson LF, Twiggs LB. Blood flow characteristics of ovarian tumours: Implications for ovarian cancer screening. Am J Obstet Gynecol 1995; 172:901-7.

41 Stein SM, Laifer-Narin S, Johnson MB, Roman LD, Muderspach LI, Tyszka JM, Ralls PW. Differentiation of benign and malignant adnexal masses: relative value of gray-scale, color Doppler, and spectral Doppler sonography. AJR Am J Roentgenol 1995; 164:381-6.

42 Zanetta G, Vergani P, Lissoni A. Color Doppler ultrasound in the preoperative assessment of adnexal masses. Acta Obstet Gynecol Scand 1994; 73:637-41

43 Prömpeler HJ, Madjar H, Sauerbrei W, Lattermann U, Pfleiderer A. Kombinierte Beurteilung von Ovarialtumoren mit transvaginaler B-Bild- und Farbdoppler-Sonographie. Geburtsh u Frauenheilk 1996;56:345-50

44 Emoto M, Udo T, Obama H, Eguchi F, Hachisuga T, Kawarabayashi T. The blood flow characteristics in borderline ovarian tumors based on both color Doppler ultrasound and histopathological analyses. Gynecol Oncol 1998; 70:351-7.

45 Tekay A, Jouppila P. Blood flow in benign ovarian tumors and normal ovaries during the follicular phase. Obstet Gynecol 1995;86:55-9.

46 Reles A, Wein U, Lübke M, Lichtenegger W. Transvaginale Farbdopplersonographie in der präoperativen Diagnostik von Adnextumoren. Geburtsh u Frauenheilk 1998;58:93-9.

47 Taylor A, Jukovic D, Bourne TH, Natucci M, Collins WP, Campbell S. Comparison of transvaginal color Doppler imaging and color Doppler energy for assessment of intraovarian blood flow. Obstet Gynecol 1998;91:561-7.

48 Nustad K, Bast RC, Obrien T, Nilszoson O, Seguin P, Suresh M, et al. Specificity and affinity of 26 monoclonal antibodies against the CA-125 antigene. First report from the ISOBM TD-1 Workshop. Tumor Biol 1996;17:175-219.
49 Hakama M, Ulf-Hacan S, Knekt P, Järvisalo J, Hakulinen T, Maatela J, Aromaa A. CA-125 a screening test for ovarian cancer. J Med Screening 1996;3:40-2

50 Malkasian GD Jr, Knapp RC, Lavin PT, Zurawski VR Jr, Podratz KC, Stanhope R, et al. Preoperative evaluation of serum CA 125 levels in premenopausal and postmenopausal patients with pelvic masses. Obstet Gynecol 1988; 71:751-6.

51 Inoue M, Fukita M, Nakazawa A, Ogawa H, Tanizawa O. Sialyl-Tn, Sialyl-Lewis XI, CA 19.9, CA 125, carcinoembryonic antigen, and tissue polypeptide antigen in differentiating ovarian cancer from benign tumors. Obstet Gynecol 1992;79:434-40.

52 Gadducci A, Ferdeghini M, Prontera C, Moretti L, Mariani G, Bianchi R, Fioretti P. The concomitant determination of different tumor markers in patients with epithelial ovarian cancer and benign ovarian masses: relevance for differential diagnosis. Gynecol Oncol 1992;44:147-54.

53 Maggino T, Gadducci A, D'Addario V, Pecorelli S, Lissoni A, Stella M, et al. Prospective multicenter study on CA 125 in postmenopausal pelvic masses. Gynecol Oncol 1994;54:117-23.

54 Schutter EM, Kenemans P, Sohn C, Kristen P, Crombach G, Westermann R, et al. Diagnostic value of pelvic examination, ultrasound, and serum CA 125 in postmenopausal women with a pelvic mass. An international multicenter study. Cancer 1994;74:1398-406.

55 Gadducci A, Baicchi U, Marrai R, Ferdeghini M, Bianchi R, Facchini V. Preoperative evaluation of D-dimer and CA 125 levels in differentiating benign from malignant ovarian masses. Gynecol Oncol 1996;60:197-202.

56 Dorum A, Kristensen GB, Abeler VM, Tropé CG, Moller P. Early detection of familial ovarian cancer. Eur J Cancer 1996;32A:1645-51.

57 Rosenthal A, Jacobs I. Ovarian cancer screening. Semin Oncol 1998;25:315-25.

58 Goldstein SR. Postmenopausal adnexal cysts: How clinical management has evolved. Am J Obstet Gynecol 1996;175:1498-501.

59 Dottino PR, Levine DA, Ripley DL, Cohen CJ. Laparoscopic management of adnexal masses in premenopausal and postmenopausal women. Obstet Gynecol 1999;93:223-8.

60 Mettler L, Jacobs V, Brandenburg K, Jonat W, Semm K. Laparoscopic management of 641 adnexal tumors in Kiel, Germany. J Am Assoc Gynecol Laparosc 2001;8:74-82.

61 Lin P, Falcone T, Tulandi T. Excision of ovarian dermoid by laparoscopy and by laparotomy. Am J Obstet Gynecol 1995;173:769-71.

62 Howard FM. Surgical management of benign cystic teratoma: laparoscopy versus laparotomy. J Reprod Med 1995;40:495-9.

63 Mais V, Ajossa S, Piras B, Marongiu D, Guerriero S, Melis GB. Treatment of nonendometriotic benign adnexal cysts: a randomized comparison of laparoscopy and laparotomy. Obstet Gynecol 1995; 86:770-4. 
64 Davison J, Park W, Penney L. Comparative study of operative laparoscopy versus laparotomy: analysis of the financial impact. J Reprod Med 1993;38:357-60.

65 Lundorff P, Thorburn J, Hahlin M, Lindblom B, Källfelt B. Adhesion formation after laparoscopic surgery in tubal pregnancy: a randomized trial versus laparotomy. Fertil Steril 1991;55:911-55.

66 Howard, Maruri F, Azzi R. Laparoscopic surgery for ectopic pregnancies: technology assessment and public health implications. Fertil Steril 1993; 59:487-98.

67 Canis M, Mage G, Pouly JL, Watthiez A, Manhes $\mathrm{H}$, Bruhat MA. Laparoscopic diagnosis of adnexal cystic masses: a 12-year experience with long term follow-up. Obstet Gynecol 1994;83:707-12.

68 SOGC. Directives concernant la laparoscopie de la masse annexielle 1998;76:1-7.

69 Kindermann G, Maassen V, Kuhn W. Laparoscopic management of ovarian tumors subsequently diagnosed as malignant: a survey from 127 German departments of obstetrics and gynecology. J Pelvic Surgery 1996;2:245-51.

70 Biran G, Golan A, Sagiv R, Glezerman M, Mencze J. Conversion of laparoscopy to laparotomy due to adnexal malignancy. Eur J Gynaecol Oncol 2002;23:157-60.

71 Jacobi CA, Sababt R, Bohn B, Zieren HU, Volk HD, Muller JM. Pneumoperitoneum with carbon dioxide stimulates growth of malignant colonic cells. Surgery 1997;121:72-8.

72 Lehner R, Wenzl R, Heinzl H, Husslein P, Sevelda P. Influence of delayed staging laparotomy after laparoscopic removal of ovarian masses later found malignant. Obstet Gynecol 1998;92:967-1.

73 Reich O, Kömetter R, Pickel H. Chronisch-vernarbende Peritonitis nach spontan rupturierter Teratomzyste - ein Fallstrick beim chirurgischen Staging von Ovarialtumoren. Geburtsh u Frauenheilk 1999;59:94-5
74 Sainz de la Cuesta R, Goff B, Fuller A, Nikrui N, Eichborn J, Rice L. Prognostic importance of intraoperative rupture of malignant ovarian epithelial neoplasms. Obstet Gynecol 1994;84:1-7.

75 Webb M, Decker D, Mussey E, Williams T. Factors influencing survival in stage I ovarian cancer. Am J Obstet Gynecol 1973;116:228.

76 Dembo AJ, Davy M, Stenwig EA, Berle EJ, Bush RS, Kjorstad K. Prognostic factors in patients with stage I epithelial ovarian cancer. Obstet Gynecol 1990;75:263-72.

77 Sevaida P, Dittrich C, Saizer H. Prognostic value of the rupture of the capsule in stage I epithelial ovarian carcinoma. Gynecol Oncol 1989; 35:321-2.

78 Sjövall K, Nilson B, Einhorn N. Different types of rupture of the tumor capsule and the impact on survival in early ovarian carcinoma. Int J Gynecol Cancer 1994;4:333-6.

79 Maiman M, Seltzer V, Boyce J. Laparoscopic excision of ovarian neoplasms subsequently found to be malignant. Obstet Gynecol 1991;77:563-5.

\section{Die Mitglieder der Arbeitsgruppe} «Adnexbefund»

Prof. Dr. med. Ekkehard Dreher, Bern; Dr. med. Markus Eberhard, Schaffhausen; PD Dr. med. Matthias Fehr, Zürich; PD Dr. med. Ossi Köchli, Zürich; Prof. Dr. med. Joachim Torhorst, Basel

\section{Leiter der Arbeitsgruppe}

Dr. med. Jean-François Delaloye, ME, Lausanne

Der Präsident der Kommission

Qualitätssicherung der SGGG

Prof. Dr. med. Urs Haller, Zürich

Der Präsident der SGGG

Dr. med. Lucio Bronz, Bellinzona 